\title{
Instantaneous damage detection of bridge structures and experimental verification
}

\author{
Serdar Soyoz ${ }^{*},+\neq$ and Maria Q. Feng ${ }^{\S}$ \\ Department of Civil and Environmental Engineering, University of California, Irvine, CA, U.S.A.
}

\begin{abstract}
SUMMARY
An extended Kalman filtering (EKF) method was developed and applied to instantaneously identify elemental stiffness values of a structure during damaging seismic events based on vibration measurement. This method is capable of dealing with nonlinear as well as linear structural responses. Identification of the structural elemental stiffness enables location as well as quantification of structural damage. The instantaneous stiffness values during an event can provide highly useful information for post-event capacity estimation. In this study, a large-scale shaking table test of a three-bent concrete bridge model was performed in order to verify the proposed damage detection method. The bridge model was shaken to different damage levels by a sequence of earthquake motions with increasing intensities. The elemental stiffness values of the structure were instantaneously identified in real time during the damaging earthquake excitations using the EKF method. The identified stiffness degradations and their locations agreed well with the structural damage observed by visual inspection and strain measurements. More importantly, the seismic response accelerations analytically simulated using the instantaneous stiffness values thus identified agreed well with the measured accelerations, demonstrating the accuracy of the identified stiffness. This study presents an experimental verification of a structural damage detection method using a realistic bridge model subjected to realistic seismic damage. Copyright (C) 2007 John Wiley \& Sons, Ltd.
\end{abstract}

KEY WORDS: extended Kalman filter; seismic damage detection; instantaneous stiffness; shaking table test; bridge structure; vibration measurement

\section{INTRODUCTION}

Sophisticated highway system in the United States is supported by tens of thousands of bridges and viaducts. Lack of information about the post-event structural integrity of these bridges can

\footnotetext{
*Correspondence to: Serdar Soyoz, Department of Civil and Environmental Engineering, University of California, Irvine, CA, U.S.A.

†'E-mail: ssoyoz@uci.edu

$\$$ Ph.D. Candidate.

${ }^{\S}$ Professor.

Contract/grant sponsor: Caltrans; contract/grant number: 59A0311
}

Copyright (C) 2007 John Wiley \& Sons, Ltd.

Received 23 April 2007

Revised 10 August 2007

Accepted 24 August 2007 
cause safety hazards to the traveling public, halt mobility of the transportation network, and disrupt emergency response. The current practice relies on visual inspection for damage detection, which is time consuming, insufficient, subjective, and requires physical presence of the crew on the structure that is potentially hazardous after major natural or man-made events such as earthquakes and accidents.

Structural condition assessment of highway bridges has long relied on visual inspection, which involves subjective judgment of inspectors and detects only local and visible flaws. The frequency of visual inspection and the qualification of the inspectors were regulated by the National Bridge Inspection Standards [1]. The Federal Highway Administration (FHWA) Recoding and Coding Guide [2] also provides guidance in terms of the condition ratings and the documentation in current practice. Even with these provisions, a recent investigation initiated by FHWA to examine the reliability of visual inspections reveals significant variability in the structural condition assignments by inspectors [3]. Moreover, visual inspection cannot quantitatively evaluate the strength and/or deformation capacity reservation of a bridge. The Long-term Bridge Performance Program (LTBP) was recently initiated by FHWA, exploring sensor-based continuous monitoring of bridges under traffic conditions as well as during extreme events [4].

Sensor-based structural health monitoring (SHM) can revolutionize the way of inspecting structures, particularly for post-event damage assessment, in a rapid, remote, automated, and objective fashion. By installing appropriate sensors at critical locations on a bridge structure, transmitting the sensor data through a communications network, and analyzing the data through a software platform, the location and severity of bridge damage caused by earthquakes or other events can be automatically, remotely, and rapidly assessed, without sending inspection crews to the site.

Dynamic characteristics of structures such as natural frequencies are widely studied for SHM and damage detection purposes. This study proposes the use of elemental stiffness of a structure as an indicator of the structural 'health' condition, based on the fact that structural damage or degradation decreases stiffness. By representing the structure with a finite element model (FEM) and measuring the structural vibration, the extent and the location of damage or degradation can be assessed by identifying the change in the elemental stiffness values. The importance of the stiffness, versus the modal parameter, identification arises from the potential that postearthquake capacity of a structure can be estimated with the knowledge of the stiffness values.

System identification methods for damage detection of structures based on vibration measurement can be found extensively in the literature (e.g. [5-9]). These methods can be grouped into two categories depending on whether the identification is carried out in frequency or time domain. The frequency domain methods basically identify the changes in modal frequencies, damping ratios, or modal shapes based on vibration measurement. Elemental stiffness values can then be further identified by minimizing the errors between the modal parameters identified from the vibration measurement and FEM simulation (e.g. [10-12]). The time-domain methods, such as the least-squares estimation (LSE), basically perform an optimization for the parameters such as stiffness and damping by directly minimizing errors between the measured and the simulated responses in time domain (e.g. [13]). However, LSE is very time consuming for online SHM and damage detection.

Kalman filtering uses not only the measurement data in a probabilistic sense but also information from structural models so that stiffness identification becomes possible even under noise-contaminated measurements and for uncertain models [14]. Results obtained by the 
extended Kalman filter (EKF) approach from simulated data with known damage scenarios were reported [15-21]. However, the applicability of the EKF approach to realistic civil engineering structures involving high uncertainties in structural modeling and loading under realistic damaging events has not yet been widely studied.

This study applies the EKF approach to identify different levels of damage on a large-scale three-bent bridge model caused by earthquake motions, by defining the damage as structural stiffness degradations. Stiffness values at the lower and upper portions of each column were selected as unknown physical parameters. The stiffness values were identified even when the structure went into nonlinear range. Also the change in their values caused by seismic damage was identified online.

\section{PROPOSED DAMAGE DETECTION METHOD AND EKF FORMULATION}

The EKF formulation, with details given in [22], can be summarized as follows, together with the proposed damage detection method.

A second-order equation of motion for a multi-degree-of-freedom (DOF) system can be expressed as

$$
M . \ddot{u}(t)+C(t) \cdot \dot{u}(t)+K(t) \cdot u(t)=-M . I . \ddot{u}_{\mathrm{g}}
$$

where $M$ is the mass matrix, $C(t)$ is the time-varying damping matrix, $K(t)$ is the time-varying stiffness matrix, $u$ is the relative displacement vector, $I$ is the influence vector, and $\ddot{u}_{\mathrm{g}}$ is the input ground acceleration. $\ddot{u}+\ddot{u}_{\mathrm{g}}$ and $\ddot{u}_{\mathrm{g}}$ are measured, $M$ is calculated from the structural design drawings, $C(t)$ is considered to be of Rayleigh-type damping, i.e. linear combination of $K(t)$ and $M$ matrices. The objective is to identify $K(t)$ which is directly used as the damage indicator.

An extended state vector is defined as

$$
x(t)=[u(t), \dot{u}(t), \Psi(t)]^{\mathrm{T}}
$$

where $\Psi(t)$ is the extended state. In this study it is the stiffness values for the lower and the upper portions of the columns.

The EKF determines the optimal estimate of the state $\hat{x}_{k \mid k}$ that minimizes the trace of the error covariance

$$
P_{k \mid k}=E\left[\left(x_{k}-\hat{x}_{k \mid k}\right)\left(x_{k}-\hat{x}_{k \mid k}\right)^{\mathrm{T}}\right]
$$

where $^{\wedge}$ denotes estimation.

There are mainly two conceptual phases in EKF, namely prediction and correction phases. In the prediction phase, state estimate $\hat{x}_{k-1 \mid k-1}$ and the error covariance $P_{k-1 \mid k-1}$ are projected ahead in time resulting in a priori estimates of $\hat{x}_{k \mid k-1}$ and $P_{k \mid k-1}$. In the correction phase, these $a$ priori estimates are filtered using the information from the new measurements resulting in $a$ posteriori estimates $\hat{x}_{k \mid k}$ and $P_{k \mid k}$.

The system can be defined as

$$
\dot{x}(t)=f(x, t)+w(t)
$$

where $w(t)$ is process noise;

$$
y(t)=h(x, t)+v(t)
$$

where $y(t)$ is the measurement and $v(t)$ is the measurement noise. 
The system is supposed to meet the following conditions:

$$
\begin{gathered}
E[w(t)]=0 \\
E\left[v_{k}\right]=0 \\
E\left[w(t) v_{k}^{\mathrm{T}}\right]=0
\end{gathered}
$$

where subscript $k$ indicates discrete time.

The values of $Q, R, x_{0}, P_{0}$ are defined as

$$
\begin{gathered}
E\left[w(t) w^{\mathrm{T}}(t)\right]=Q_{c}(t) \\
E\left[v_{j} v_{k}^{\mathrm{T}}\right]=R_{k} \delta_{j k} \\
E\left[x\left(t_{0}\right)\right]=x_{0} \\
E\left[\left(x\left(t_{0}\right)-x_{0}\right)\left(x\left(t_{0}\right)-x_{0}\right)^{\mathrm{T}}\right]=P_{0}
\end{gathered}
$$

where subscript ' $c$ ' indicates continuous time and $j$ indicates discrete time.

Linearized measurement matrix $H_{k}$ (for steps 3-5) can be obtained as

$$
\begin{gathered}
H(x, t)=\frac{\partial h(x, t)}{\partial x} \\
H_{k}=H\left(\hat{x}_{k \mid k-1}, t_{k}\right)
\end{gathered}
$$

Linearized state matrix $F_{k}$ (for calculating the state transition matrix) can be obtained as

$$
\begin{aligned}
& F(x, t)=\frac{\partial f(x, t)}{\partial x} \\
& F_{k}=F\left(\hat{x}_{k \mid k-1}, t_{k}\right)
\end{aligned}
$$

State transition matrix $\Phi_{k-1}$ can be obtained (for step 1) as

$$
\Phi_{k-1}=I+\int_{t_{k-1}}^{t_{k}} F\left(\hat{x}_{t \mid k-1}, t\right) \Phi\left(t, t_{k-1}\right) \mathrm{d} t
$$

The last equation is expressed as

$$
\Phi\left(t_{k}, t_{k-1}\right)=\exp \left(\Delta t . F_{k-1}\right), \quad \text { Constant } F \forall t \in\left[t_{k-1}, t_{k}\right)
$$

This is approximated as

$$
\Phi\left(t_{k}, t_{k-1}\right)=I+\Delta t \cdot F_{k-1}
$$

Also, the process noise can be discretized as

$$
Q_{k-1}=\Delta t \cdot Q_{c}\left(t_{k-1}\right)
$$


After initializing $Q, R, x_{0}, P_{0}$ in Equations (9)-(12), Kalman filtering can be performed in the following five steps:

Step 1: Predicting covariance:

$$
P_{k \mid k-1}=\Phi_{k-1} P_{k-1 \mid k-1} \Phi_{k-1}^{\mathrm{T}}+Q_{k-1}
$$

Step 2: Predicting state:

$$
\hat{x}_{k \mid k-1}=\Phi_{k-1} \hat{x}_{k-1 \mid k-1}
$$

Step 3: Computing Kalman gain:

$$
G_{k}=P_{k \mid k-1} H_{k}^{\mathrm{T}}\left(H_{k} P_{k \mid k-1} H_{k}^{\mathrm{T}}+R_{k}\right)^{-1}
$$

Step 4: Correcting covariance:

$$
P_{k \mid k}=\left(I-G_{k} H_{k}\right) P_{k \mid k-1}
$$

Step 5: Correcting state:

$$
\hat{x}_{k \mid k}=\hat{x}_{k \mid k-1}+G_{k}\left[y_{k}-H_{k} \hat{x}_{k \mid k-1}\right]
$$

Therefore, state vector $x$ is obtained at each time step. As described in Equation (2), the state vector contains information of not only displacement and velocity but also of the stiffness value. This means that the stiffness value is identified for each time step.

\section{SHAKING TABLE TEST}

In order to investigate the effectiveness of the proposed damage detection method based on identification of stiffness change using vibration measurement and the EKF approach, shaking table tests were performed on a large-scale three-bent bridge model. The model was shaken to different levels of damage by a sequence of earthquake ground motions with increasing intensities. Using the response acceleration measurement, elemental stiffness values at the specified locations of the model were identified.

\subsection{Bridge model}

The experiment was performed on a three-bent reinforced concrete bridge model as shown in Figure 1. Each of the three bents was supported separately on a shaking table. The bridge deck consisted of three post-tensioned beams. Each of the bents has two columns. All the columns had the same design cross sections, but the bents were of different heights leading to different transverse stiffness. To resemble the inertia of other parts of the superstructure not built into the model, or the inertia from the abutments on both ends, additional masses were added, each consisted of 60 kips concrete blocks and 26.5 kips blocks. The shaking tables were driven by input acceleration in the transverse direction. The gaps were provided at the connections between the deck and the bents, so that the deck was divided into two simply supported spans in the vertical direction. However, in the transverse direction the existence of the post-tension tendons and the pre-stressed strain made the three individual beams behave as one beam with a large cross section. Eleven FBA-11 (Kinemetrics Inc.)-type accelerometers were used to obtain the vibration response of the bridge model in the transverse direction, with their locations indicated in Figure 1. 


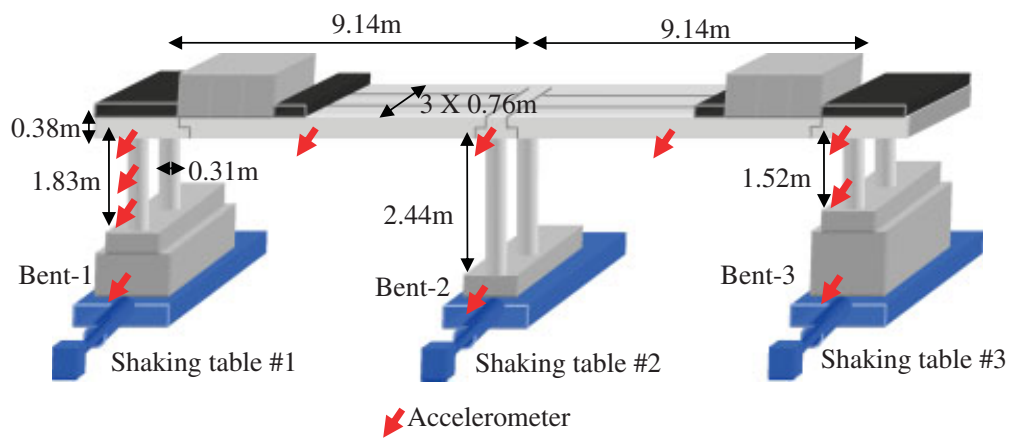

Figure 1. Schematic view of the bridge model and sensor layout.

\subsection{Test procedure}

Strong ground input motions were classified into different levels, such as low, moderate, high, severe, and extreme levels. In between these strong motions, low amplitude white noise is introduced to perturb the model in the corresponding damage level. Figure 2 shows the time history of the input motions. Strong motion records are $20 \mathrm{~s}$ long, while the white-noise records are $60 \mathrm{~s}$ long. All the time history records have $200 \mathrm{~Hz}$ sampling frequency. Figure 3 shows the frequency content of the input motions. Strong motion records contain energy between 0 and $10 \mathrm{~Hz}$ while white noise contains energy up to $30 \mathrm{~Hz}$. Table I lists the sequence of the tests including seismic and white-noise excitations and the peak ground acceleration (PGA) of the inputs. Three shaking tables were driven by the same signal to produce coherent input. Different levels of damage were observed on the bridge after each strong ground motion. Damage description shown in Table I represents the visual observation during the experiment. This damage scenario is confirmed by the strain measurement using strain gauges embedded in steel rebars.

During the test, after each strong motion, cracks were marked and photos were taken to document the damage. Some examples are shown in Figures 4 and 5. Due to different transverse stiffness of the bents, dynamic behavior was highly dominated by the torsion-demanding high transverse movement for the first and the third bent. This effect becomes clearer from the modal shapes presented in the following section. This explains the reason of the severe damage on these two and comparatively lighter damage on the second, middle, bent.

The observed damage can be traced using strain measurements. Figure 6 shows time history of strain measured by strain sensors embedded in steel rebars at the bottom of the left column of Bent 3 during the Test-14. From the strain time history, it is observed that drift occurred approximately at $20 \mathrm{~s}$, indicating yielding of the steel rebars. Although strain monitoring at appropriate locations can directly provide seismic damage information as demonstrated in Figure 6, it is difficult to embed strain sensors in a reinforced concrete bridge during the bridge construction and it is impossible to do so for an existing bridge. It, therefore, was the goal of this research to identify structural damage based on vibration measurement using surface-attached accelerometers.

\section{VERIFICATION OF DAMAGE DETECTION METHOD}

This section first presents the identification of modal parameters of the bridge from its acceleration responses to the white-noise inputs. Because the level of shaking is low, the 

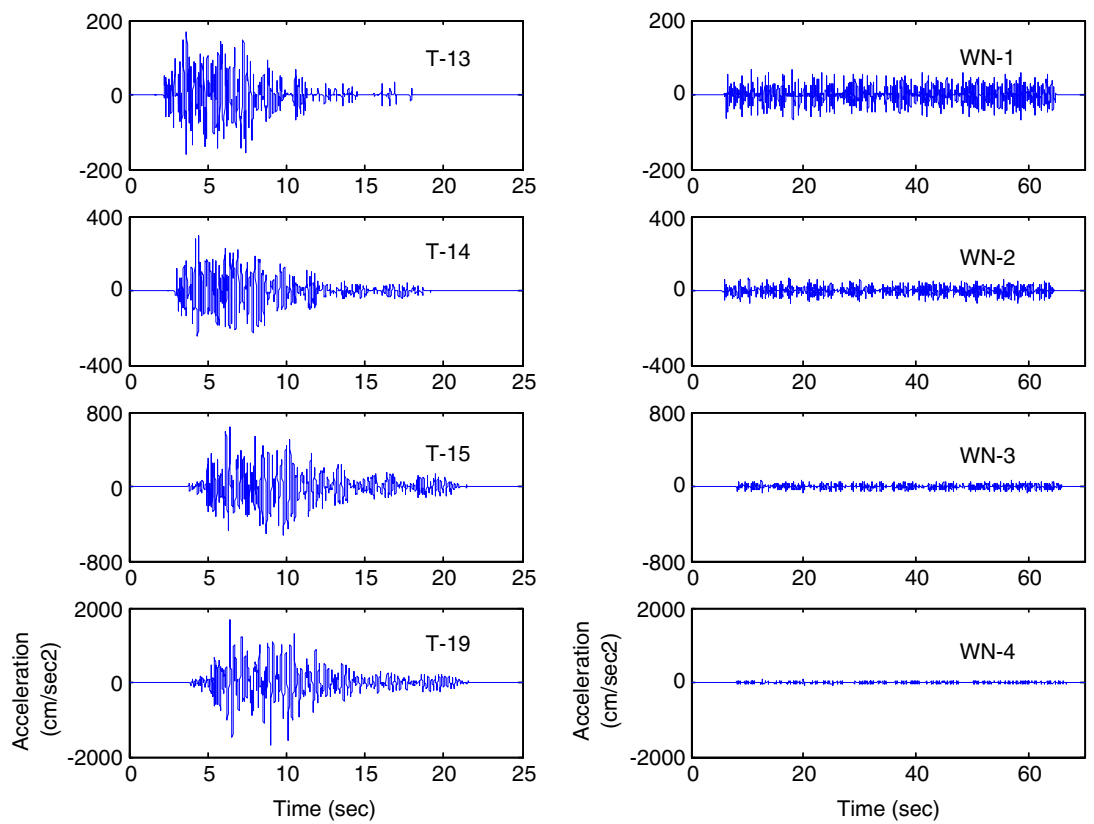

Figure 2. Time histories of the input motions.
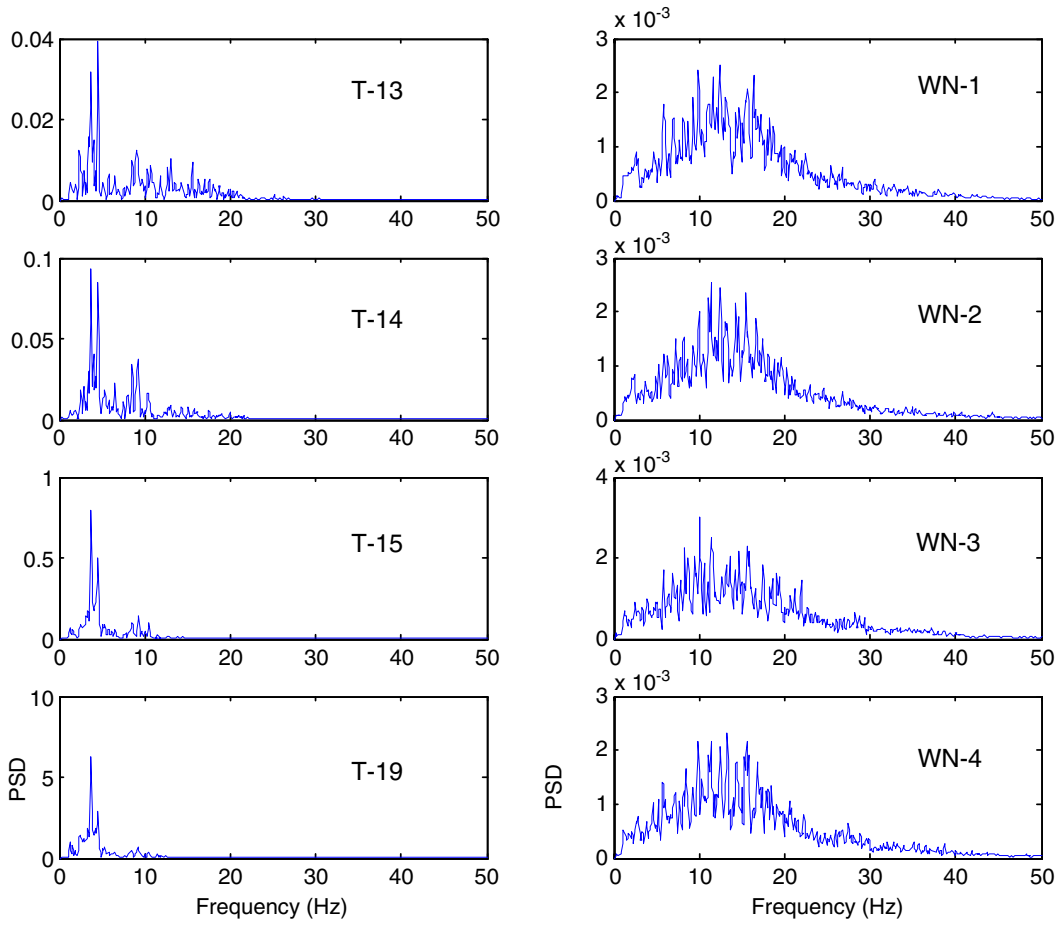

Figure 3. Power spectral densities of the input motions. 
Table I. Test procedure.

\begin{tabular}{lccc}
\hline Test & Ground motion description & PGA $(\mathrm{g})$ & Damage description \\
\hline WN-1 & White noise in transverse & 0.07 & \\
T-13 & Low earthquake in transverse & 0.17 & Bent-1 yields \\
T-14 & Moderate earthquake in transverse & 0.32 & Bent-3 yields \\
WN-2 & White noise in transverse & 0.07 & Bent-2 yields \\
T-15 & High earthquake in transverse & 0.63 & \\
WN-3 & White noise in transverse & 0.07 & Bent-3 steel buckles \\
T-19 & Extreme earthquake in transverse & 1.70 & \\
WN-4 & White noise in transverse & 0.07 & \\
\hline
\end{tabular}

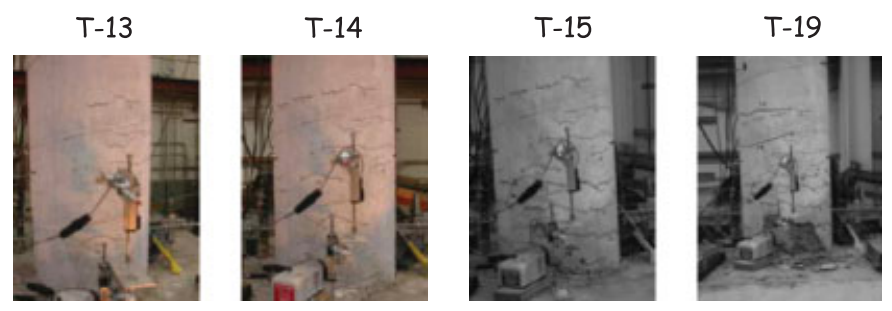

Figure 4. Damage observed on Bent-1 after each test.

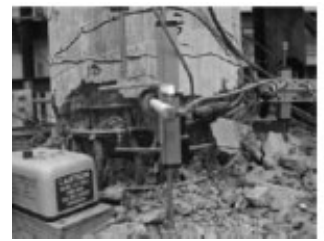

Lower portion

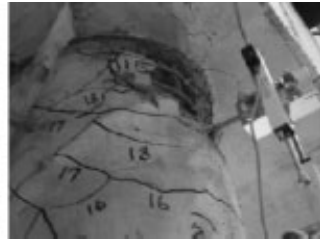

Upper portion

Figure 5. Damage observed on the lower and upper portions of Bent-3 after T-19.

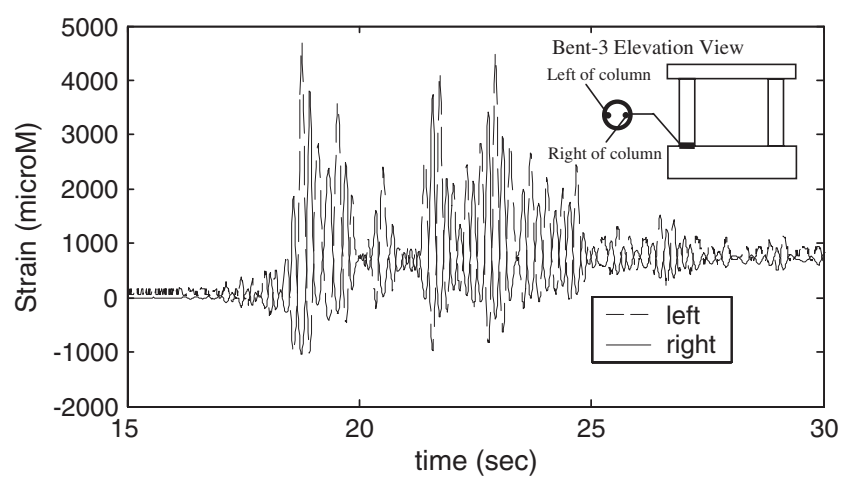

Figure 6. Strain measurements at Bent-3 during T-14. 
response can be treated as linear and thus the classic Fourier transform method can be employed. Then structural stiffness values at selected locations of the bridge columns were instantaneously identified during the seismic event, using the EKF method presented in Section 2. The accuracy of the identified stiffness was verified by the agreement between the acceleration responses measured and obtained by EKF. Finally, a comparison of the modal frequencies resulting from the two methods is also presented.

\subsection{Modal identification based on white-noise excitation}

White-noise inputs have low amplitudes so they cause no further damage to the structure. As a result, the Fourier transform can be used to obtain the modal frequencies of the bridge model at each damage level. Figure 7 plots the power spectral density of the average response acceleration recorded by all the 11 sensors under each of the white-noise excitations. From the shift of the modal frequencies under WN-1 and WN-2, the effect of yielding can be clearly seen. Another observation is that after T-14, the second mode $(3.7 \mathrm{~Hz})$ and third mode $(13.7 \mathrm{~Hz})$ are no longer visible. Figure 8 shows the modal shapes obtained under WN-1. The shift of modal frequencies indicates damage, but provides no information about the locations of the damage. It is also difficult to quantify the extent of damage from the modal frequencies or shapes. Therefore, it was the goal of this research to identify the change in structural elemental stiffness by utilizing the time-domain EKF method.

\subsection{FEM and calibration}

An FEM was generated in MATLAB using the stick element and lumped mass approach, which is later employed in the EKF algorithm. Figure 9 shows the DOFs assigned to the model, each of which has both translational and rotational DOFs. DOFs 1,2, 7, 9, 10, 11, 13, and 5 are at the same locations/directions as the sensors; DOFs 8 and 12 were created so that the additional deck mass added to the bridge model in the experiment could be taken into consideration. DOFs 3,4 , and 6 were assigned so that the lower and upper portions of each column could be treated separately as an extended state for the EKF.

As the first step, the stiffness values for the FEM (based on design drawings of the bridge) were updated using the measured bridge response to the white-noise excitation $\mathrm{WN}-1$ before any damage. The modal frequencies and shapes measured under the WN-1 excitation and obtained from the updated FEM agreed well, as shown in Table II, demonstrating the accuracy of the FEM. In this study, mass is assumed to be accurately estimated based on the design drawings and does not change due to damage. Error in the mass estimation will obviously cause error in the natural frequencies, particularly the higher frequencies. Considering the fact that the bridge response is mainly dominated by lower frequencies (less than $5 \mathrm{~Hz}$ ), the variation in mass is considered less important (in comparison with stiffness). Afterwards, acceleration responses to WN-1 were simulated by this updated model and compared with the measured responses, by which the damping values in the FEM were updated. It was found that the Rayleigh damping model used in the FEM was satisfactory.

\subsection{Damage detection results based on EKF}

This calibrated model was employed in the EKF method, which was then used to identify the elemental stiffness of the bridge model during seismic (rather than white noise) excitations. It is 


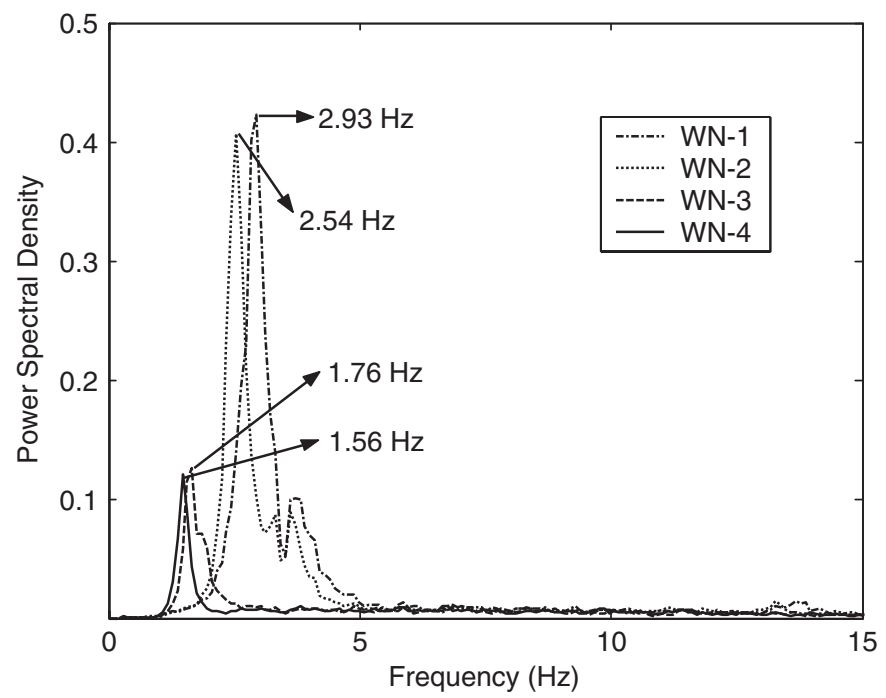

Figure 7. Modal frequencies obtained from responses to white-noise inputs.

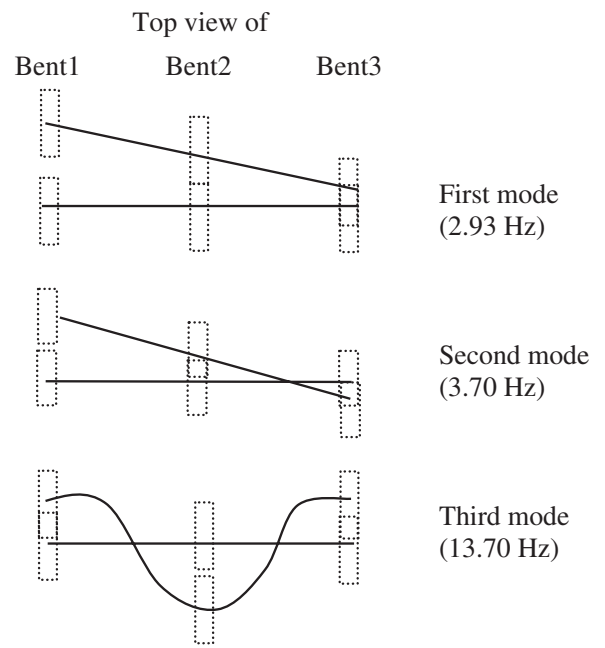

Figure 8. Modal shapes obtained from responses to $\mathrm{WN}-1$ input.

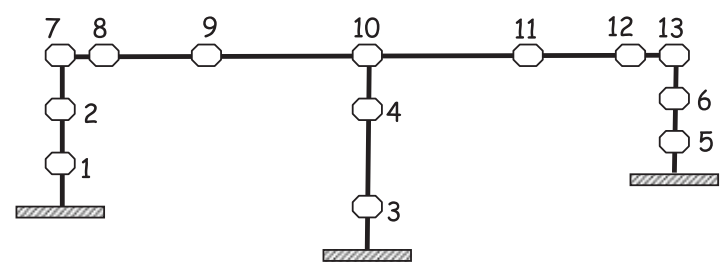

Figure 9. Schematic view of FEM. 
Table II. FEM calibration.

\begin{tabular}{lcccccr}
\hline & & \multicolumn{5}{c}{ Shape (DOF) } \\
\cline { 3 - 7 } & Frequency (Hz) & 7 & 9 & 10 & 11 & 13 \\
\hline $\begin{array}{l}\text { Model } \\
\text { Experimental (WN-1) }\end{array}$ & 2.93 & 1.00 & 0.83 & 0.68 & 0.53 & 0.34 \\
$\begin{array}{l}\text { Analytical (FEM) } \\
\text { Mode2 }\end{array}$ & 2.95 & 1.00 & 0.85 & 0.67 & 0.51 & 0.31 \\
$\begin{array}{l}\text { Experimental (WN-1) } \\
\text { Analytical (FEM) }\end{array}$ & 3.70 & 1.00 & 0.61 & 0.30 & -0.05 & -0.43 \\
Mode3 & 3.69 & 1.00 & 0.62 & 0.31 & -0.08 & -0.40 \\
Experimental (WN-1) & & & & & & \\
Analytical (FEM) & 13.70 & -0.79 & 0.61 & 1.00 & 0.61 & -0.79 \\
\hline
\end{tabular}
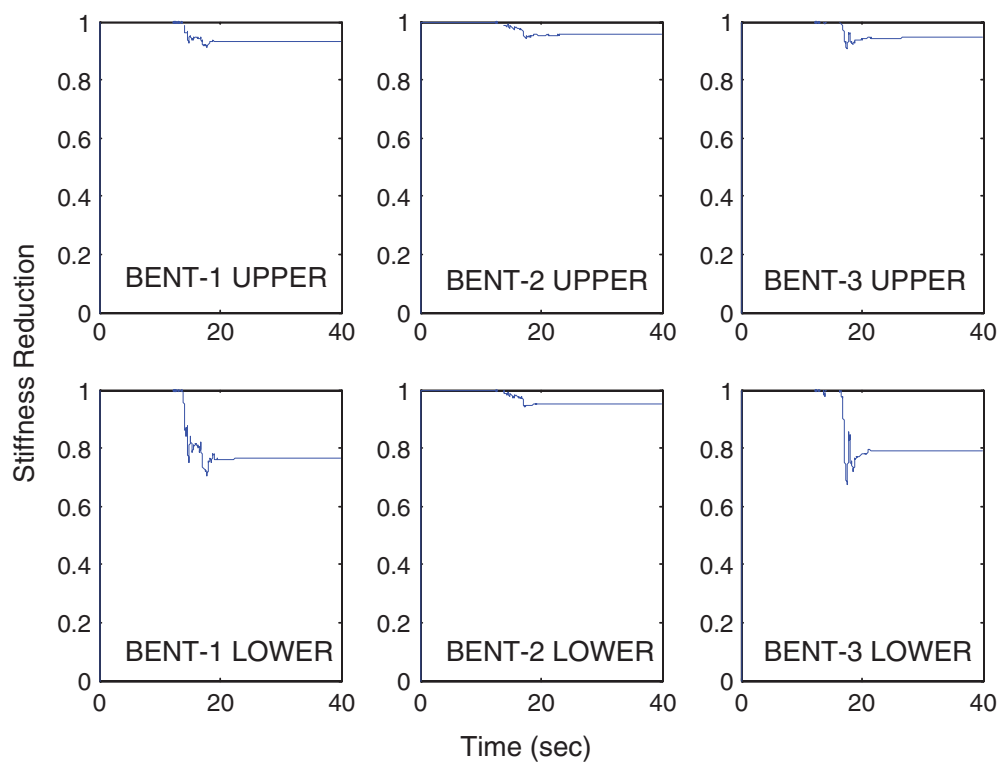

Figure 10. Identified instantaneous stiffness during T-13.

noted that the proposed EKF method is applicable to nonlinear as well as linear responses, which is a significant advantage. Stiffness values in the damaged zones are considered as piecewise linear within each time step. However, they are updated at each time step, so that the nonlinearity of the stiffness is taken into consideration. These identified stiffness values at each time step are referred to as instantaneous stiffness values. At each time step, the state transition matrix in Equation (17) is obtained analytically from the FEM, each of the five steps of EKF shown in Equations (21)-(25) is implemented, the structural stiffness is corrected after Equation (25), and the new Jacobian matrix, Equations (15) and (16) (which are used for the state transition matrix), is calculated again. Hence, at the end of the seismic input motion, the 


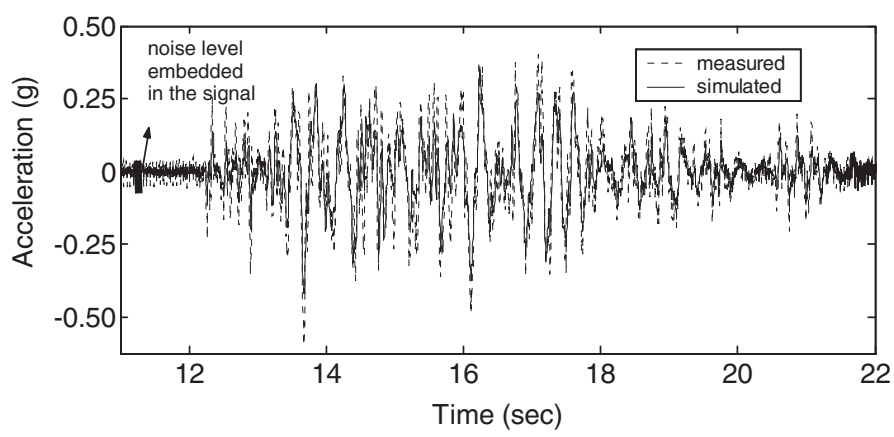

Figure 11. Comparison of measured and simulated responses to T-13.

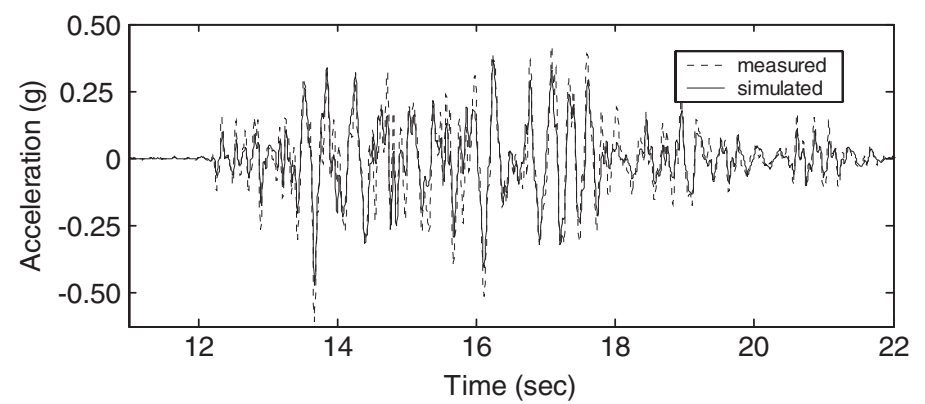

Figure 12. Comparison of measured and simulated responses to T-13 after filtering.

structural stiffness value at assigned locations can be obtained. Figure 10 shows the time histories of the identified stiffness values at different locations during seismic excitation T-13. The stiffness value is presented by the ratio between the identified current stiffness versus the original stiffness before damage.

Figure 11 compares the time histories of the response acceleration at DOF 10 to seismic excitation T-13 measured by the accelerometer and obtained by EKF. It is noted that the measured acceleration during T-13 contains a high-level noise. Upon filtering the measured response with the butter-worth-type low-pass filter of $20 \mathrm{~Hz}$, an excellent agreement between the measured and simulated response accelerations can be obtained, as shown in Figure 12. The excellent agreement confirms the accuracy of the identified instantaneous stiffness values during the event.

Similarly, the instantaneous stiffness values, updated each time step, during the T-14, T-15 and T-19 seismic excitations were identified, as shown in Figures 13-15, respectively. Significant degradation of the stiffness at the lower portion of Bent- 1 can be observed from the identified stiffness time histories in Figure 13. This is consistent with the damage observed by the strain measurement and visual inspection, as described in Table I. Furthermore, the agreement between measured and simulated acceleration responses, as shown in Figure 16, during T-19 confirms the accuracy of these instantaneously identified stiffness values.

Furthermore, the post-event modal frequencies are computed by FEM analysis using the stiffness values identified by EKF method. These modal frequency values are compared with those measured from the responses to the low-level white-noise input after the event. As 

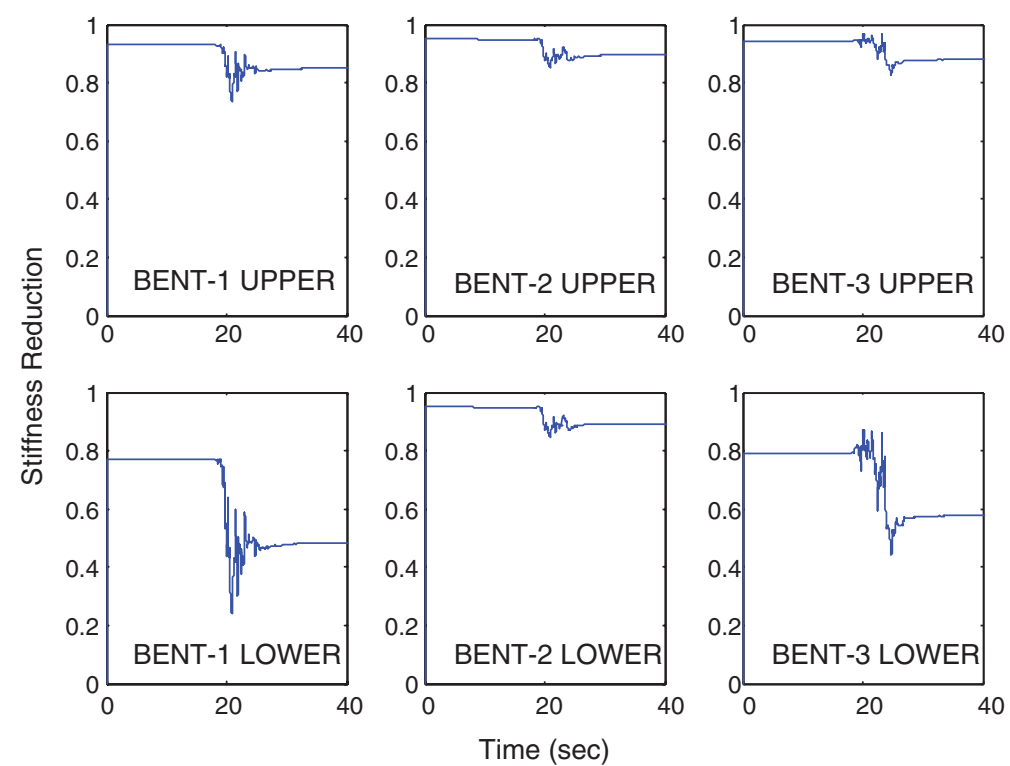

Figure 13. Identified instantaneous stiffness during T-14.
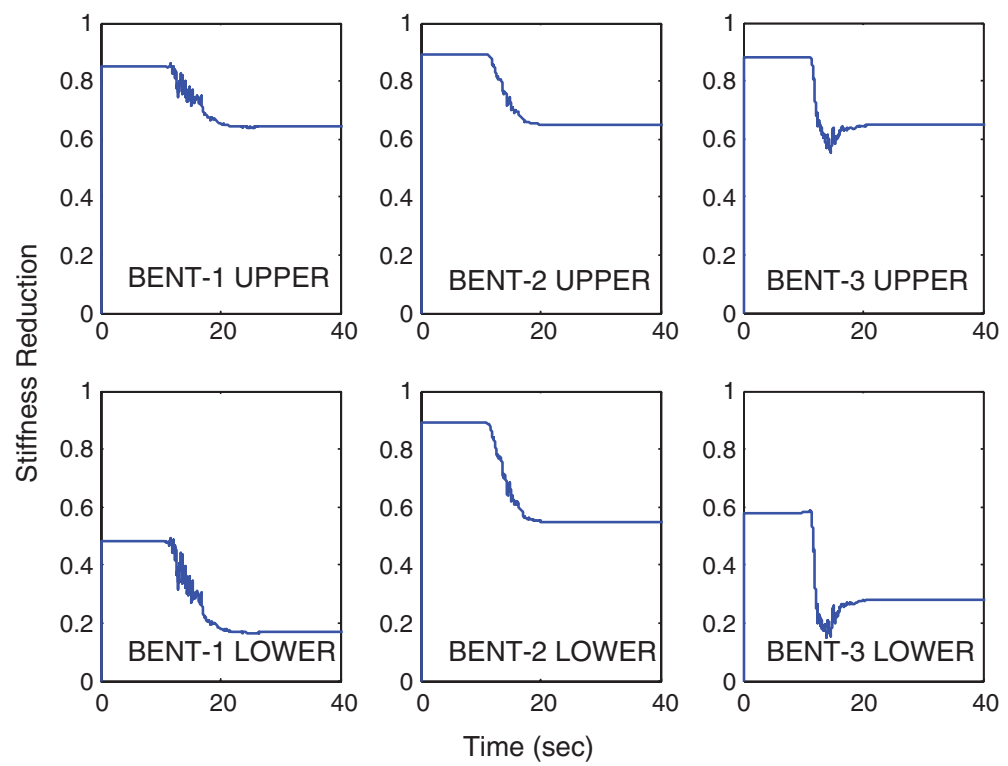

Figure 14. Identified instantaneous stiffness during T-15.

demonstrated in Table III, modal frequencies obtained by two different approaches are quite close to each other. A possible reason for the slight discrepancy might be due to concrete cracking all over the bridge model other than the only plastic hinges as considered in the FEM and EKF formulations. 

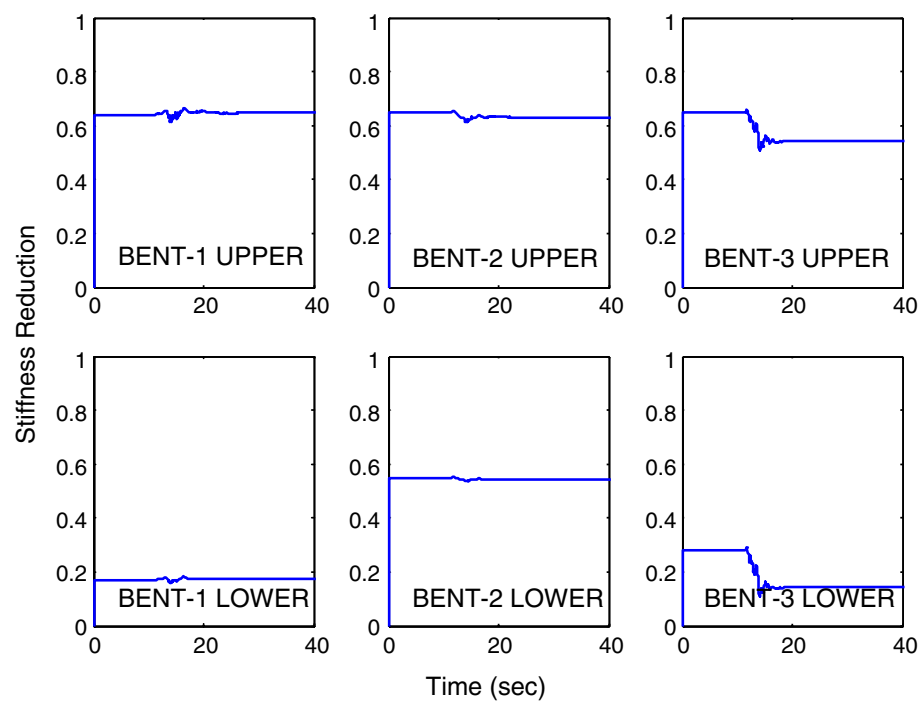

Figure 15. Identified instantaneous stiffness during T-19.

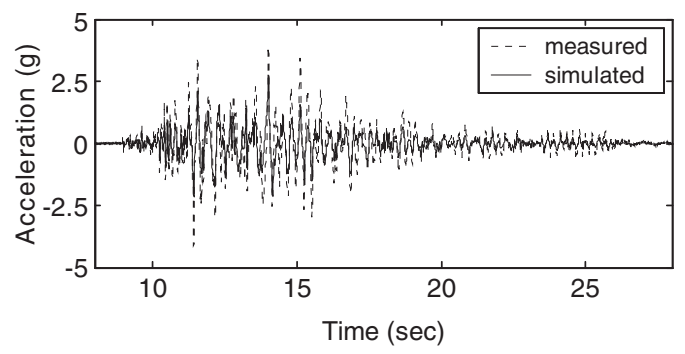

Figure 16. Comparison of measured and simulated responses to T-19.

Table III. Comparison of computed and measured first modal frequencies.

\begin{tabular}{l|c|cc}
\hline Input & Measured $(\mathrm{Hz})$ & & Computed $(\mathrm{Hz})$ \\
\hline $\mathrm{WN}-1$ & 2.93 & $\longrightarrow$ & 2.94 \\
\hline $\mathrm{T}-13$ & & & 2.74 \\
\hline $\mathrm{T}-14$ & & & 2.55 \\
\hline $\mathrm{WN}-2$ & 2.54 & & \\
\hline $\mathrm{T}-15$ & & & 1.82 \\
\hline $\mathrm{WN}-3$ & 1.76 & & \\
\hline $\mathrm{T}-19$ & & & \\
\hline $\mathrm{WN}-4$ & 1.56 & & \\
\hline
\end{tabular}




\section{CONCLUSION}

This paper presents the development of extended Kalman filtering (EKF) method for instantaneously identifying elemental stiffness values of a structure during a damaging earthquake event based on vibration measurement, together with experimental verification using a large-scale realistic bridge model subjected realistic seismic damage. Significant advantages of this time-domain EKF method include its ability to deal with nonlinear as well as linear responses. The following conclusions can be made through the shaking table verification test:

1. The EKF method is capable of identifying and tracing the change of structural elemental stiffness values during a damaging earthquake event in real time.

2. The acceleration responses to the earthquake excitation analytically simulated using the stiffness values thus identified agreed well with the measured acceleration responses, demonstrating the accuracy of the identified stiffness values, as well as the analytical model.

3. The effectiveness of the EKF method was further demonstrated by comparing the postevent modal frequency identified by the EKF method with that measured from the postevent white-noise (ambient) vibration.

4. The identified stiffness degradations and their locations agree well with seismic damage observed by visual inspection and strain measurement. Identification of stiffness degradation based on acceleration measurement enables the location and quantification of structural damage.

The instantaneous stiffness of a structure during an earthquake event, identified by the proposed EKF method, can be highly useful for the estimation of post-event capacity of structures. This is the subject of the authors' future study.

\section{ACKNOWLEDGEMENTS}

This study is sponsored by Caltrans, under the research grant 59A0311. The authors are thankful for insightful comments from project manager $\mathrm{Mr}$ Li-Hong Sheng. The shaking table experiments were conducted at University of Nevada, Reno in conjunction with Dr Saidii's and Sander's NSF-NEES project. The authors appreciate the technical discussions and comments from Prof. Faryar Jabbari, Department of Mechanical and Aerospace Engineering and Dr Yangbo Chen, Department of Civil and Environmental Engineering at the University of California Irvine.

\section{REFERENCES}

1. National Bridge Inspection Standards (NBIS). Code of Federal Regulations, No. 23CFR650. U.S. Government Printing Office: Washington, DC, 1996; 238-240.

2. Federal Highway Administration (FWHA). Recording and Coding Guide for the Structure Inventory and Appraisal of the Nation's Bridges. U.S. Department of Transportation, Washington, DC, 1995.

3. Phares BM, Washer GA, Rolander DD, Graybeal BA, Moore M. Routine highway bridge inspection condition documentation accuracy and reliability. Journal of Bridge Engineering 2004; 9(4):403-413.

4. http://www.tfhrc.gov/structur/ltbp.htm

5. Ghanem R, Shinozuka M. Structural system identification. I: theory. Journal of Engineering Mechanics 1995; 121(2):255-264.

6. Shinozuka M, Ghanem R. Structural system identification. II: experimental verification. Journal of Engineering Mechanics 1995; 121(2):265-273. 
7. Safak E. Identification of linear structures using discrete-time filters. Journal of Structural Engineering 1991; 17(10):3064-3085.

8. Safak E. Adaptive modeling, identification and control of dynamic structural systems. I: theory. Journal of Engineering Mechanics 1989; 115(11):2386-2405.

9. Yoshimoto R, Mita A, Okada K. Damage detection of base-isolated buildings using multi-inputs multi-outputs subspace model identification. Earthquake Engineering and Structural Dynamics 2005; 34:307-324.

10. Feng MQ, Kim JM. Identification of a dynamic system using ambient vibration measurements. Journal of Applied Mechanics 1998; 65(2):1010-1023.

11. Feng MQ, Bahng EY. Damage assessment of jacketed RC columns using vibration tests. Journal of Structural Engineering 1999; 125(3):265-271.

12. Feng MQ, Kim DK, Yi JH, Chen YB. Baseline models for bridge performance monitoring. Journal of Engineering Mechanics 2003; 131(5):562-569.

13. Soyoz S, Feng MQ, Safak E. Real-time structural health monitoring incorporating soil-structure interaction. Hundredth Anniversary Earthquake Conference, San Francisco, CA, 18-22 April 2006.

14. Kalman RE. A new approach to linear filtering and prediction problems. Journal of Basic Engineering 1960; 82(1):35-45.

15. Yun CB, Shinozuka M. Identification of nonlinear structural dynamic systems. Journal of Structural Mechanics 1980; 8:187-203.

16. Hoshiya S, Saito E. Structural identification by extended Kalman filter. Journal of Engineering Mechanics 1984; 110(12):1757-1770.

17. Yang JN, Lin S, Huang H, Zhou L. An adaptive extended Kalman filter for structural damage identification. Structural Control and Health Monitoring 2006; 13(4):849-867.

18. Straser EG, Kiremidjian AS. Monitoring and evaluating civil structures using measured vibration. Proceedings of 14th International Modal Analysis Conference, Dearborn, MI, U.S.A., 1996; 84-90.

19. Loh CH, Chung ST. A three-stage identification approach for hysteretic systems. Earthquake Engineering and Structural Dynamics 1993; 22:129-150.

20. Loh CH, Tou IC. A system identification approach to the detection of changes in linear and non-linear structural parameters. Earthquake Engineering and Structural Dynamics 1995; 24:85-97.

21. Ghanem R, Ferro G. Health monitoring for strongly non-linear systems using the ensemble Kalman filter. Structural Control and Health Monitoring 2006; 13(1):245-259.

22. Grewal MS, Andrews AP. Kalman Filtering Theory and Practice Using Matlab. Wiley: New York, 2001. 